Interventions undertaken to develop pan-London clinical priorities

Development of London Clinical Network (LCN) Delivery Model using improvement science principles.

Development of weighted criteria for pan-London action

Framework developed on the role of the Regional Medical team

Clinical strategy event for senior professionals and clinical leaders to consider the issues and priorities which should form the basis of a renewed vision and strategy.

Using its Delivery model the LCN will be the primary delivery vehicle working alongside key stakeholders, 2019/20 delivery plans have been developed for priority areas. Improvements will be measured through reporting on indicators to Programme Boards through the Regional Medical Director.

The successful use of science to develop a strategy for action by bringing wider stakeholders together promoting a collaborative approach and galvanising commitment from the wider quality improvement architecture to work jointly to drive improvement.

System wide leadership brings local benefits, reduced duplication, enables shared learning and better use of resources. Trust and a commitment to collaborative working are required to bring about impactful change.

\section{IMPROVING CARDIOVASCULAR DISEASE (CVD) PREVENTION IN LONDON; A CO-ORDINATED APPROACH}

H Al-Omari, H Cutting, C Thomson. London Clinical Network, NHS England and Improvement, London, UK

\subsection{6/leader-2019-FMLM.45}

Issue Multiple agencies interested in improving Cardiovascular Disease (CVD) prevention were working in silos, resulting in repeated approaches to CCGs offering a variety of solutions in partnership with different organisations, causing confusion and limited outcomes.

Intervention London CCGs requested the Cardiac and Stroke Clinical Networks to develop a unified approach. The Networks engaged improvement organisations forming the London Cardiovascular Disease Prevention Partnership (CVDPP) to achieve this for Atrial Fibrillation, Hypertension and Familial Hypercholesterolaemia. Condition specific steering groups were formed to provide clinical, patient and system leadership and support delivery of improvement projects.

Strategy for improvement The CVDPP held workshops attended by numerous system partners to develop a London vision for CVD prevention, share best practice, include patients and discuss solutions to common barriers.

A RACI was used to clarify roles of organisations engaged in CVD prevention. Detailed plans for each condition are being developed with STPs using a logic model and menu of evidence-based interventions. Agreed detection and treatment targets for each condition to be achieved by 2023.

Impact

1. Increased detection and management of CVD risk factors;

2. Additional 400 lives saved each year by reducing heart attacks and strokes;
3. Empowering Londoners to take control of their circulatory health.

Lessons learnt Working collaboratively and innovatively across organisations with competing priorities brings complexities. Implementing large scale change across a complex and varied landscape requires patience, flexibility and time coupled with a clear aim.

Messages for others Working in a co-ordinated partnership minimises duplication and maximises utilisation of limited resources to achieve large scale change. Multiple and sometimes conflicting approaches to commissioners has been eliminated through partnership working.

\section{IMPROVING WEEKEND MEDICAL WARD COVER AT ROYAL SURREY COUNTY HOSPITAL}

Mengfei Lu, Kishan Moosai, Roselle Herring. Royal Surrey County Hospital, UK

\subsection{6/leader-2019-FMLM.46}

Introduction A 7-day NHS service is proven to achieve greatest benefits for patients as published by recent evidence based review. Despite the limitation of NHS resources, healthcare professionals should aim to provide the best quality of care to patients at all times.

Concerns about weekend ward cover, especially for medical patients on outlier wards, were raised through emails and verbal feedback from doctors and nursing teams. This project aims to improve clinical effectiveness for medical patients over the weekend.

Methods Qualitative data was collected through verbal and written feedbacks from wards, managers and medical teams.

A new poster with up-to-date bleep numbers for medical doctors over the weekend was designed, and a morning weekend handover was established together with collection of bleeps in a designated area. These changes were communicated to doctors, site managers, nursing and administrative staff and switchboard.

Results Numerous factors which were contributing to the problem have been identified:

1. Incorrect bleep numbers were recorded on the switchboard system

2. The use of bleeps were inconsistent amongst doctors

3. The ward staffs were unaware of the bleep numbers that were carried by the doctors

4. Uncertainty of which doctors are on-call

Conclusion Several implementations have been introduced as a result to improve communication between ward staffs and oncall doctors.

1. Designated buddying outlier wards for specific medical wards.

2. Designated bleeps numbers for doctors covering specific wards, all bleeps need to be returned to EAU office at the end of the shift.

3. Up-to-date on-call board on EAU

4. Laminated A4 medical on-call bleep sheet on every ward to guide ward staffs to bleep the accurate doctors.

5. Up-to-date list of bleeps with switchboard

6. Emails to all medical consultants, trainees, ward team and site managers with updates of the implementation. 